\title{
TEOREMA DE REORDENAMENTO DE SÉRIES DE
}

\author{
RIEMANN
}

\author{
RIEMANN'S SERIES REARRANGEMENT THEOREM
}

\section{AMANDA SIQUEIRA $^{\mathrm{a}}$ GERSON G. LEDESMA ${ }^{\mathrm{b}}$}

\begin{abstract}
Resumo
Neste trabalho irá ser feita uma demonstração explícita do Teorema de Reordenamento de Riemann, afirma-se que se tivermos uma série condicionalmente convergente é possível fazê-la convergir para qualquer número desejado, reordenando convenientemente seus termos. Conseguinte a introdução do Teorema de Abel para vermos alguns exemplos de reordenamento da série harmônica alternada.
\end{abstract}

Palavras-chave: Séries, Reordenamento, Teorema de Riemann.

\begin{abstract}
In this work we will give an explicit demonstration of Riemann's Rearrangement Theorem, which tells us that if we have a conditionally convergent series it is possible to make it converge to any desired number by conveiently rearrangement of its terms. We will introduce Abel's Theorem to see some examples of the rearrangement of the alternating harmonic series
\end{abstract}

Keywords: Series, Rearrangement, Riemann's Theorem.

MSC2020: 40A05

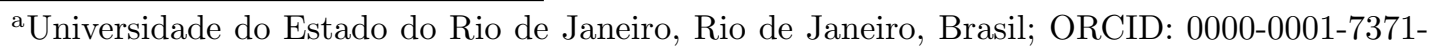
9938 E-mail: 1amanda.siqueira@gmail.com

${ }^{\mathrm{b}}$ Universidade do Estado do Rio de Janeiro, Rio de Janeiri, Brasil; ORCID:0000-0002-8351-7456 E-mail: guido.ledesma@ime.uerj.br 


\section{Introdução}

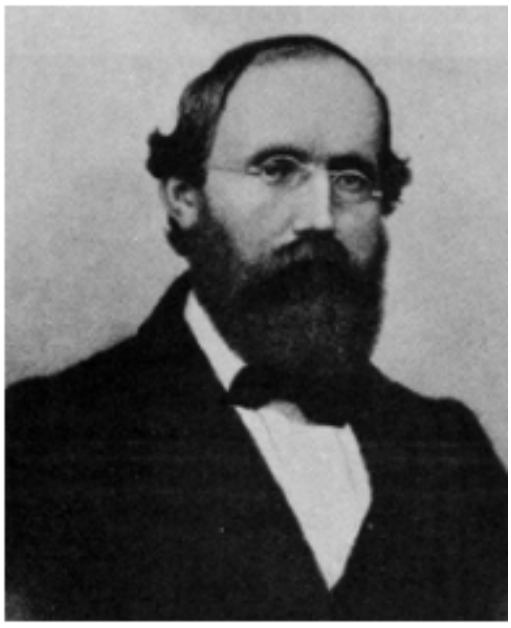

Figura 1: Bernhard Riemann Fonte://www.svpwiki.com/GeorgFriedrich-Bernhard-Riemann

Em 1827, Peter Lejeune-Dirichlet descobriu que a soma de uma série pode ser alterada, reordenando seus termos enquanto trabalhava em condições que garantiam a convergência das séries de Fourier [2]. Ele foi o primeiro a notar que os termos de certas séries poderiam ser reorganizados de forma a resultar uma soma diferente a soma da série original, mas não foi capaz de mostrar porque isso era possível.

Posteriormente, Bernhard Riemann começou a trabalhar em um artigo que ampliava os resultados de Dirichlet sobre a convergência das séries de Fourier. Ele logo encontrou uma explicação notável para esse comportamento, descobrindo assim que isso funciona para qualquer série condicionalmente convergente. Esse resultado ficou conhecido como "O Teorema de Reordenamento de Riemann" em seu artigo da série de Fourier.

Embora o trabalho tenha sido concluído, no final de 1853, ele não foi publicado até sua morte, em 1866, sob o título "Uber die Darstellbarkeit einer Function durch eine trigonometrische Reihe" (Sobre a representação de uma função por séries trigonométricas) [7]. Estes acontecimentos são explicados em mais detalhes no artigo [5].

\section{Preliminares}

Neste artigo considera-se a reta real estendida que resulta de adicionar à reta de números reais os elementos $+\infty$ e $-\infty$ e será denotada por $\overline{\mathbb{R}}$, isto é,

$$
\overline{\mathbb{R}}=\mathbb{R} \cup\{+\infty\} \cup\{-\infty\} .
$$

Os elementos $+\infty$ e $-\infty$ podem ser relacionados com os números reais através de certas operações aritméticas e uma relação de ordem. Para detalhes sobre as propriedades aritméticas da reta estendida recomendamos ao leitor ver [10]. Só chamamos atenção para as somas $+\infty+(-\infty)$ e $-\infty+(+\infty)$, que não são definidas em $\overline{\mathbb{R}}$. Sendo assim, a reta estendida não é mais um corpo. Com relação ao produto em $\overline{\mathbb{R}}$, se $a=0$, define-se: $+\infty \cdot a=a \cdot(+\infty)=0$ e $-\infty \cdot a=a \cdot(-\infty)=0$, o que não 
segue a convenção usual vista no contexto de limites, porém são definições úteis em estudos mais avançados de Matemática.

A reta estendida torna-se um conjunto totalmente ordenado se definirmos a ordem:

$$
-\infty<a<+\infty \quad \forall a \in \mathbb{R} \text {. }
$$

Outra propriedade importante da reta estendida é que todo subconjunto $S$ de $\overline{\mathbb{R}}$ tem um ínfimo (definido como a maior cota inferior do conjunto) e um supremo (definido como a menor cota superior do conjunto), ao contrário do que acontece na análise clássica, em que para um conjunto ter ínfimo (supremo) este deve ser não vazio e limitado inferiormente (superiormente). No caso de reta estendida, se $E \subseteq \overline{\mathbb{R}}$ não for limitado inferiormente (superiormente), então

$$
\inf E=-\infty(\sup E=+\infty) \text {. }
$$

O caso curioso acontece quando o conjunto for vazio, pois teríamos que

$$
\inf \emptyset=+\infty \quad \text { e } \quad \sup \emptyset=-\infty
$$

Isto pode ser explicado notando que todo número real (por vacuidade) é uma cota inferior do conjunto vazio, assim segue da Definição de ínfimo que inf $\emptyset=+\infty$. $O$ outro caso é explicado de forma similar.

A topologia usual considerada na reta estendida, é a topologia gerada pela família $\mathcal{F}$ constituída por todos os conjuntos da forma:

$(r,+\infty]=\{x \in \mathbb{R}, x>r\} \cup\{+\infty\} \quad$ e $\quad[-\infty, r)=\{x \in \mathbb{R}, x<r\} \cup\{-\infty\}, \quad r \in \mathbb{R}$

Ou seja, os abertos desta topologia, além do conjunto vazio e $\overline{\mathbb{R}}$, são todas as interseções finitas de conjuntos pertencentes à família $\mathcal{F}$ e todas as uniões arbitrárias destas interseções finitas. A família $\mathcal{F}$ é uma sub-base da topologia (ver [3]). Os conjuntos $(r,+\infty]$ serão vizinhanças abertas do elemento $+\infty$ e os conjuntos $[-\infty, r)$ serão vizinhanças abertas do elemento $-\infty$, com $r \in \mathbb{R}$. Observamos que para $a<b, a, b \in \mathbb{R}$, intervalo aberto $(a, b)$ é dado pela interseção $(a,+\infty] \cap[-\infty, b)$. Como $\mathbb{R} \subset \overline{\mathbb{R}}$, a topologia usual da reta será a topologia induzida pela topologia da reta estendida.

Definição 2.1. $\quad$ 1. Uma sequência na reta estendida é uma função $x: \mathbb{N} \rightarrow \overline{\mathbb{R}}$ usualmente denotada por $\left\{x_{n}\right\}$ ou $\left(x_{n}\right)$. O $x_{n}$ é chamado de termo n-ésimo. 
2. Uma sequência $\left\{x_{n}\right\}$ na reta estendida $\overline{\mathbb{R}}$ é dita convergente a um elemento $x \in \overline{\mathbb{R}}$, se uma das situações abaixo ocorre:

(a) Se $x \in \mathbb{R}$ e dado um $\epsilon>0$, existe $n_{0} \in \mathbb{N}$ tal que se

$$
n \geq n_{0} \quad \Rightarrow \quad\left|x_{n}-x\right|<\epsilon .
$$

Quando isso acontecer, denotaremos por $\lim _{n \rightarrow+\infty} x_{n}=x$ ou $\lim x_{n}=x$ ou $x_{n} \rightarrow x$.

(b) Se $x=+\infty$ e dado $M<+\infty$, existe $n_{0} \in \mathbb{N}$ tal que se

$$
n \geq n_{0} \quad \Rightarrow \quad x_{n}>M
$$

Neste caso escrevemos $\lim x_{n}=+\infty$.

(c) Se $x=-\infty$ e dado $m>-\infty$, existe $n_{0} \in \mathbb{N}$ tal que se

$$
n \geq n_{0} \quad \Rightarrow \quad x_{n}<m \text {. }
$$

Neste caso escrevemos $\lim x_{n}=-\infty$

As seguintes definições foram tiradas de [6]

Definição 2.2. Seja $\left\{x_{n}\right\}$ uma sequência e seja $\left\{n_{j}\right\}$ uma sequência de inteiros positivos, tal que $k_{1}<k_{2}<k_{3}<\ldots$. A sequência

$$
\left\{x_{k_{n}}\right\}_{n=1}^{+\infty}
$$

é chamada de subsequência de $\left\{x_{n}\right\}$.

Definição 2.3. $\quad$ 1. Seja $F$ um subconjunto de $\mathbb{R}$. Diremos que $x \in \mathbb{R}$ é um ponto de aderência de $F$ se, somente se, para todo $r>0$ verifica-se

$$
I_{r}(x) \cap F \neq \emptyset
$$

em que $\left.I_{r}(x)=\right] x-r, x+r[$. O conjunto de todos os pontos de aderência de $F$ é chamado de fecho de $F$ e será denotado por $\bar{F}$. Um conjunto é chamado de fechado se, e somente se, este coincide com seu fecho.

2. Um número $x \in \mathbb{R}$ é chamado de ponto de acumulção de $F$, se

$$
I_{r}^{*}(x) \cap F \neq \emptyset, \quad \forall r>0
$$


em que $I_{r}^{*}(x)=I_{r}(x) \backslash\{x\}$. O conjunto de todos os pontos de acumulação de $F$ é chamado de conjunto derivado de $F$ e será denotado por $F^{\prime}$.

Observação 2.1. Verifica-se que $\bar{F}=F \cup F^{\prime}$, ver [6].

Definição 2.4. Uma sequência é chamada de limitada se existe um $K \in \mathbb{R}^{+}$tal que

$$
\left|x_{n}\right| \leq K, \forall n \in \mathbb{N}
$$

Teorema 2.1. (Bolzano-Weierstrass) Toda sequência limitada na reta possui subsequência convergente.

Demonstração. Ver [6].

Observação 2.2. Note que no contexto de reta estendida, dado uma sequência $\left\{x_{n}\right\}$ não limitada, então esta possui uma subsequência $\left\{x_{k_{n}}\right\}$ que converge $a+\infty$ ou $-\infty$. Daqui podemos ter uma versão do Teorema de Bolzano-Weierstrass para reta estendida.

Teorema 2.2. Toda sequência na reta estendida possui uma subsequência convergente.

Demonstração. Seja $\left\{x_{n}\right\}$ uma sequência na reta estendida, assim temos duas possibilidades, $\left\{x_{n}\right\}$ é limitada ou não. Se $\left\{x_{n}\right\}$ for limitada, então, pelo Teorema 2.1, está possui subsequência convergente. Agora, se $\left\{x_{n}\right\}$ não for limitada, para cada $n \in \mathbb{N}$ existe um $k_{n} \in \mathbb{N}$ tal que

$$
\left|x_{k_{n}}\right|>n
$$

Daqui, construímos uma subsequência $\left\{x_{k_{n}}\right\} \subseteq\left\{x_{n}\right\}$ e definimos o conjunto

$$
A=\left\{k_{n}: x_{k_{n}}>0\right\} \subseteq \mathbb{N}
$$

Este conjunto $A$ pode ser finito ou infinito. Se $A$ for infinito, então obtemos uma subsequência $\left\{x_{l_{n}}\right\}$ tal que

$$
\lim _{n \rightarrow+\infty} x_{l_{n}}=+\infty
$$

Se $A$ é finito, então o complementar deste é infinito assim podemos obter uma subsequência $\left\{x_{s_{n}}\right\}$ tal que

$$
\lim _{n \rightarrow+\infty} x_{s_{n}}=-\infty
$$

Portanto, toda sequência na reta estendida possui uma subsequência convergente. 
Definição 2.5. Dado uma sequência $\left\{x_{n}\right\}$ na reta estendida, definimos o conjunto

$$
E=\left\{\alpha \in \overline{\mathbb{R}}: \exists\left\{x_{k_{n}}\right\} \subseteq\left\{x_{n}\right\} \text { tal que } x_{k_{n}} \rightarrow \alpha\right\}
$$

Este é chamado de conjunto dos limites subsequenciais de $\left\{x_{n}\right\}$.

Exemplo 2.1. Vamos achar os limites subsequenciais, da sequência $\left\{x_{n}\right\}$ definida por

$$
x_{n}=\left\{\begin{array}{l}
0, \quad n=3 k+1 \\
-n, \quad n=3 k+2 \\
n, \quad n=3 k
\end{array}\right.
$$

A subsequência $\left\{x_{3 k+1}\right\}=\{0\}$ converge para $0,\left\{x_{3 k+2}\right\}=\{-3 k+2\}$ converge a $-\infty$ e a subsequência $\left\{x_{3 k}\right\}=\{3 k\}$ converge $a+\infty$. Logo, $E=\{0,+\infty,-\infty\}$.

Observação 2.3. Em $\overline{\mathbb{R}}$, segue do Teorema 2.2, que $E \neq \emptyset$ para qualquer sequência na reta estendida.

No que segue do trabalho usaremos a notação de [8].

Definição 2.6. Seja $\left\{x_{n}\right\}$ uma sequência na reta estendida. Seja E o conjunto de limites subsequenciais de $\left\{x_{n}\right\}$. Definimos

$$
s^{*}=\sup E \quad e \quad s_{*}=\inf E .
$$

Os números $s^{*}$ e $s_{*}$ são chamados de limites superior e inferior de $\left\{x_{n}\right\}$, respectivamente, e usamos a notação,

$$
\limsup _{n \rightarrow+\infty} x_{n}=s^{*} \quad e \quad \liminf _{n \rightarrow+\infty} x_{n}=s_{*}
$$

O seguinte resultado exibe a relação entre lim inf, lim sup e lim de uma sequência.

Teorema 2.3. Uma sequência $\left\{x_{n}\right\}$ na reta estendida converge se, e somente se,

$$
\liminf _{n \rightarrow+\infty} x_{n}=\limsup _{n \rightarrow+\infty} x_{n}=\lim _{n \rightarrow+\infty} x_{n}
$$

Demonstração. Ver [8]

Lema 2.1. Seja $\left\{x_{n}\right\}$ uma sequência na reta real e $E$ o conjunto de seus limites subsequênciais. Então, dado $y \in E \cap \mathbb{R}, \epsilon>0$ e $l \in \mathbb{N}$, existe um $m \in \mathbb{N}$ tal que

$$
m>l \quad e \quad\left|x_{m}-y\right|<\epsilon
$$


Demonstração. Dado $y \in E, \epsilon>0$ e $l \in \mathbb{N}$. Como $y \in E$, então existe uma subsequência $\left\{x_{k_{n}}\right\}$ tal que

$$
x_{k_{n}} \rightarrow y
$$

Pela convergência existe $n_{0} \in \mathbb{N}$ tal que

$$
n \geq n_{0} \quad \Rightarrow \quad\left|x_{k_{n}}-y\right|<\epsilon \text {. }
$$

Pela definição de subsequência existe um $n$ suficientemente grande tal que

$$
n>n_{0} \quad \text { e } \quad k_{n}>l
$$

Então, tomando $m=k_{n}$, temos

$$
m>l \quad \text { e }\left|x_{m}-y\right|<\epsilon .
$$

Teorema 2.4. Os limites subsequenciais de uma sequência $\left\{x_{n}\right\}$ na reta estendida formam um subconjunto fechado de $\mathbb{R}$.

Demonstração. Seja E o conjunto de todos os limites subsequenciais de $\left\{x_{n}\right\}$. Queremos provar que E é fechado, para isso precisamos provar que $E=\bar{E}$. Sabemos que $E \subseteq \bar{E}$ sempre é verdade, então resta provar que $\bar{E} \subseteq E$. Suponha que $q \in \bar{E}$. Basta considerar o caso quando o $q$ é ponto de acumulação de $E$. Assim precisamos construir uma subsequência de $\left\{x_{n}\right\}$ que converge para $q$.

Se $q=+\infty$, como $q \in \bar{E}$, segue que existe uma sequência $\left\{q_{n}\right\} \subseteq E$ tal que

$$
q_{n} \rightarrow q
$$

Como estamos considerando $q$ um ponto de acumulação, $q_{n} \neq+\infty$, para todo $n \in \mathbb{N}$, assim, pelo Lema 2.1 , existe um $k_{n} \in \mathbb{N}$ tal que

$$
\left|x_{k_{n}}-q_{n}\right|<\frac{1}{n}
$$

e, além disso, estes $\left\{k_{n}\right\}$ podem ser escolhidos verificando

$$
k_{1}<k_{2}<k_{3}<\ldots
$$


Assim, de (1), a subsequência $\left\{x_{k_{n}}\right\}$ de $\left\{x_{n}\right\}$ verifica que

$$
q_{n}-\frac{1}{n}<x_{k_{n}} \Rightarrow \lim _{n \rightarrow+\infty} x_{k_{n}}=+\infty
$$

Logo, $q \in E$. De forma similar pode-se provar que se $q=-\infty$ então $q \in E$.

Suponha que $q \in E \cap \mathbb{R}$, assim escolhemos $n_{1} \in \mathbb{N}$ tal que $x_{n_{1}} \neq q$, e chamemos de $\delta=\left|q-x_{n_{1}}\right|$. Como q é um ponto de acumulação de $E$, então $I_{\frac{\delta}{4}}^{*}(q) \cap E \neq \emptyset$, portanto existe um $y_{1} \in I_{\frac{\delta}{4}}^{*}(q) \cap E$. Como $y_{1} \in E$, então pelo Lema 2.1 existe $n_{2}>n_{1}$ tal que

$$
\left|y_{1}-x_{n_{2}}\right|<\frac{\delta}{4}
$$

Então

$$
\left|q-x_{n_{2}}\right| \leq\left|q-y_{1}\right|+\left|y_{1}-x_{n_{2}}\right|<\frac{\delta}{4}+\frac{\delta}{4}=\frac{\delta}{2} .
$$

Agora suponha que temos construído os índices $n_{1}, n_{2}, \ldots, n_{i-1}$, como q é um ponto de acumulação de E, existe um $y_{i} \in I_{\frac{\delta}{2^{i}}}^{*}(q) \cap E$. Como $y_{i} \in E$, pelo Lema 2.1, existe um $n_{i}>n_{i-1}$ tal que

$$
\left|y_{i}-x_{n_{i}}\right|<\frac{\delta}{2^{i}}
$$

Então

$$
\left|q-x_{n_{i}}\right| \leq\left|q-y_{i}\right|+\left|y_{i}-x_{n_{i}}\right|<\frac{\delta}{2^{i}}+\frac{\delta}{2^{i}}=\frac{\delta}{2^{i-1}}
$$

Portanto

$$
\left|q-x_{n_{i}}\right|<\frac{\delta}{2^{i-1}}, \quad i=1,2,3 \ldots
$$

Como temos que $2^{1-i} \delta \rightarrow 0$, quando $i \rightarrow+\infty$, então $x_{n_{i}} \rightarrow q$. Portanto, $q \in$ E.

Teorema 2.5. Seja $\left\{s_{n}\right\}$ uma sequência na reta estendida. Seja E e $s^{*}$ como na Definição 2.6. Então $s^{*}$ tem as seguintes propriedades:

(a) $s^{*} \in E$.

(b) Seja $x \in \overline{\mathbb{R}}$, se $x>s^{*}$, existe um inteiro $N$ tal que $n>N$ implica $s_{n}<x$.

Além disso, s* é o único elemento na reta estendida com essas propriedades.

Demonstração. Antes de iniciar a demonstracão, note que a propriedade $(a)$ nos diz que o limsup da sequência é um limite subsequencial e a propriedade (b) nos diz que existem apenas um número finito de elementos acima de qualquer número maior que o limsup. Agora, vamos fazer a prova deste Teorema. 
(a) Se $s^{*}=+\infty$, e como $s^{*}=\sup E$, então E não é limitado superiormente. Daí, $\left\{s_{n}\right\}$ não é limitado superiormente, pois caso contrário existiria um $M>0$ tal que

$$
s_{n} \leq M, \quad \forall n \in \mathbb{N}
$$

Em particular, se temos $z \in E$, então existe uma subsequência $\left\{s_{k_{n}}\right\} \subseteq\left\{s_{n}\right\}$ tal que

$$
s_{k_{n}} \rightarrow z
$$

Assim temos que $s_{k_{n}} \leq M$, para todo $n \in \mathbb{N}$ e aplicando limite

$$
\lim _{n \rightarrow+\infty} s_{k_{n}} \leq \lim _{n \rightarrow+\infty} M \Rightarrow z \leq M
$$

Como $z \in E$ é arbitrário então E é limitado superiormente, o que gera uma contradição. Assim, $\left\{s_{n}\right\}$ não é limitado superiormente, portanto, para cada $n \in \mathbb{N}$, existe $k_{n} \in \mathbb{N}$ tal que

$$
s_{k_{n}}>n \text {. }
$$

Daqui, dado $M>0$, pela propriedade Arquimediana, existe um $n_{o} \in \mathbb{N}$ tal que $M<n_{0}$. Assim, se $n \geq n_{0}$, então $n>M$. Logo,

$$
s_{k_{n}}>M
$$

e portanto,

$$
\lim _{n \rightarrow+\infty} s_{k_{n}}=+\infty
$$

Logo, encontramos uma subsequência $\left\{s_{k_{n}}\right\} \subseteq\left\{s_{n}\right\}$ tal que $s_{k_{n}} \rightarrow+\infty$, portanto $+\infty \in E$.

Se $s^{*}$ é um número real, então E é limitado superiormente. Temos que $s^{*}=$ $\sup E$, então $s^{*} \in \bar{E}$. Pelo Teorema 2.4 temos que E é fechado e portanto $s^{*} \in E$.

Se $s^{*}=-\infty$, então $\left\{s_{n}\right\}$ é limitado superiormente, pois se $\left\{s_{n}\right\}$ não fosse limitado superiormente, então conseguiríamos uma subsequência $\left\{s_{k_{n}}\right\} \subseteq\left\{s_{n}\right\}$ tal que

$$
s_{k_{n}} \rightarrow+\infty
$$

e, portanto, $+\infty \in E$, o que é um absurdo, pois $\sup E=-\infty$. Chamemos de 
$M>0$ a cota superior de $\left\{s_{n}\right\}$. Afirmamos que

$$
\lim _{n \rightarrow+\infty} s_{n}=-\infty
$$

Com efeito, dado $m<0$ definamos o seguinte conjunto

$$
A=\left\{n \in \mathbb{N}: s_{n} \in[m, M]\right\} \subseteq \mathbb{N} .
$$

Existem duas possibilidades em que A é finito ou A é infinito. Suponha que A é infinito e então enumerando e ordenando o conjunto A temos,

$$
A=\left\{k_{1}, k_{2}, \ldots\right\}
$$

Assim, temos a subsequência $\left\{s_{k_{m}}\right\}$ de $\left\{s_{n}\right\}$ tal que

$$
m \leq s_{k_{m}} \leq M
$$

Assim, $\left\{s_{k_{m}}\right\}$ é limitado e segue do Teorema de Bolzano-Weierstrass, que existe $\left\{s_{l_{m}}\right\} \subseteq\left\{s_{k_{m}}\right\}$ tal que

$$
s_{l_{m_{k}}} \rightarrow s
$$

E portanto $s \in E$, o que é um absurdo, pois $\sup E=-\infty$. Logo A é finito. Então, existe um $n_{0} \in \mathbb{N}$ tal que se

$$
n \geq n_{0} \quad \Rightarrow \quad s_{n}<m \text {. }
$$

Logo,

$$
\lim _{n \rightarrow+\infty} s_{n}=-\infty
$$

Portanto, $-\infty \in E$.

(b) Note que esta propriedade é verdadeira por vacuidade no caso $s^{*}=+\infty$. Vamos fazer a prova para o caso $s^{*}<+\infty$. Se $x=+\infty$, como $s^{*}<+\infty$, segue que a sequência $\left\{s_{n}\right\}$ é limitada, logo

$$
s_{n}<x, \quad \forall n \geq 1
$$

Por outro lado, se $x$ for um número real procedemos pelo absurdo, isto é, suponha que exista um número $x>s^{*}$ tal que $s_{n} \geq x$ para infinitos valores de 
n. Enumerando este, temos uma subsequência $\left\{s_{m_{n}}\right\}$ tal que

$$
x \leq s_{m_{n}}, \quad \forall n \in \mathbb{N} .
$$

Temos que $\left\{s_{m_{n}}\right\}$ é limitado superiormente, pois caso contrário existiria uma subsequência $\left\{s_{l_{m_{n}}}\right\} \subseteq\left\{s_{m_{n}}\right\}$ tal que

$$
s_{l_{m_{n}}} \rightarrow+\infty
$$

o que implicaria que $+\infty \in E$, gerando uma contradição. Então, $\left\{s_{m_{n}}\right\}$ é uma sequência limitada e pelo Teorema Bolzano-Weierstrass possui uma subsequência convergente, isto é, existe $\left\{s_{k_{m_{n}}}\right\} \subseteq\left\{s_{m_{n}}\right\}$ e $y \in \mathbb{R}$ tal que

$$
s_{k_{m_{n}}} \rightarrow y
$$

Por (3) verifica-se que $y \geq x$, o que implica $y>s^{*}$, contrariando a definição de $s^{*}$, pois $y \in E$.

Agora, vamos mostrar que estas propriedades caracterizam o limite superior da sequência. Se temos um $\bar{s} \in \overline{\mathbb{R}}$ verificando (a) e (b) vamos mostrar que

$$
\bar{s}=s^{*} .
$$

Com efeito, como $\bar{s} \in E$, então

$$
\bar{s} \leq s^{*} \quad \Rightarrow \quad \bar{s}<s^{*} \text { ou } \bar{s}=s^{*} .
$$

Suponha que $\bar{s}<s^{*}$. Se $\bar{s}=+\infty$, então segue que $s^{*}=+\infty, \operatorname{logo} \bar{s}=s^{*}$, contradizendo a suposição. Assim $\bar{s}<+\infty$, logo existe um $k \in \mathbb{R}$ tal que

$$
\bar{s}<k<s^{*}
$$

por (b), existe um $N \in \mathbb{N}$ tal que

$$
\text { se } n \geq N \quad \Rightarrow \quad s_{n}<k \text {. }
$$

Assim, só existe uma quantidade finita de elementos da sequência a direita do 
$k$, de onde $s^{*} \notin E$, o que gera uma contradição. Logo,

$$
\bar{s}=s^{*} .
$$

Teorema 2.6. Seja $\left\{s_{n}\right\}$ uma sequência na reta estendida. Seja E e $s_{*}$ como na Definição 2.6. Então $s_{*}$ tem as seguintes propriedades:

(a) $s_{*} \in E$.

(b) Seja $x \in \overline{\mathbb{R}}$, se $x<s_{*}$, existe um inteiro $N$ tal que $n>N$ implica $s_{n}>x$.

Além disso, $s_{*}$ é o único elemento na reta estendida com essas propriedades.

Demonstração. A prova é de forma análoga ao Teorema 2.5.

\section{Teorema de Reordenamento de Riemann}

As ideias desenvolvidas nesta seção são baseadas nos livros [8] e [4].

Definição 3.1. Dado uma sequência de números reais $\left\{x_{n}\right\}$, escrevemos o objeto formal

$$
\sum_{n=1}^{+\infty} x_{n} \text { ou simplesmente } \sum x_{n}
$$

e chamamos de série. A série converge, se a sequência $\left\{s_{k}\right\}$ definida por

$$
s_{k}=\sum_{n=1}^{k} x_{n}=x_{1}+x_{2}+\ldots+x_{k}
$$

converge. Os números $s_{k}$ são chamados de somas parciais.

Definição 3.2. A série $\sum x_{n}$ converge absolutamente, se a série $\sum\left|x_{n}\right|$ é convergente. Caso a série seja convergente e não seja, absolutamente convergente, dizemos que a série é condicionalmente convergente.

Lema 3.1. Se $\sum a_{n}$ for uma série condicionalmente convergente, então a série formada pelos termos positivos e a série formada pelo módulo dos termos negativos são divergentes.

Demonstração. Seja, $\sum_{n=1}^{+\infty} p_{n}$ formado pelos termos positivos de $\sum a_{n}$ e seja $\sum_{n=1}^{+\infty} q_{n}$ formada pelos valores absolutos dos termos negativos de $\sum a_{n}$. Isto é,

$$
p_{n}=\frac{\left|a_{n}\right|+a_{n}}{2} \quad \text { e } \quad q_{n}=\frac{\left|a_{n}\right|-a_{n}}{2}, \forall n \in \mathbb{N} .
$$


Se ambas fosse convergentes, então

$$
\sum\left(p_{n}+q_{n}\right)=\sum\left|a_{n}\right|
$$

convergiria, contrariando a hipótese. Suponha que $\sum p_{n}$ fosse convergente e $\sum q_{n}$ divergente,

$$
\sum_{n=1}^{N} q_{n}=\sum_{n=1}^{N} p_{n}-\sum_{n=1}^{N} a_{n}
$$

o que implicaria na convergência de $\sum q_{n}$, contradizendo a suposição. O mesmo acontecerá se $\sum q_{n}$ convergente e $\sum p_{n}$ divergente. Portanto, ambas divergem, isto é

$$
\sum_{n=1}^{+\infty} p_{n}=+\infty \quad \text { e } \quad \sum_{n=1}^{+\infty} q_{n}=+\infty
$$

Teorema 3.1. (Reordenamento de Riemann) Seja $\sum a_{n}$ uma série condicionalmente convergente e seja

$$
-\infty \leq \alpha \leq \beta \leq+\infty
$$

Então, existe um reordenamento $\sum a_{n}^{\prime}$ com somas parciais $s_{n}^{\prime}$ tal que,

$$
\liminf _{n \rightarrow+\infty} s_{n}^{\prime}=\alpha \quad e \quad \limsup _{n \rightarrow+\infty} s_{n}^{\prime}=\beta
$$

Demonstração. Sejam $P_{1}, P_{2}, P_{3}, \ldots$ os termos não negativos de $\sum a_{n}$, na ordem que eles acontecem, e $Q_{1}, Q_{2}, Q_{3}, \ldots$ são os valores absolutos dos termos negativos de $\sum a_{n}$, também na ordem original. As séries $\sum P_{n}$ e $\sum Q_{n}$ diferem de $\sum p_{n}$ e $\sum q_{n}$, definidas no Lema 3.1, apenas pelos termos zeros, portanto elas são divergentes.

Construiremos $\left\{m_{n}\right\}$ e $\left\{k_{n}\right\}$ sequências tais que o reordenamento da forma

$$
P_{1}+\ldots+P_{m_{1}}-Q_{1}-\ldots-Q_{k_{1}}+P_{m_{1}+1}+\ldots+P_{m_{2}}-Q_{k_{1}+1}-\ldots-Q_{k_{2}}+\ldots
$$

satisfaz (4). Para isto, escolheremos sequências $\left\{\alpha_{n}\right\}$ e $\left\{\beta_{n}\right\}$, tal que $\alpha_{n} \rightarrow \alpha$, $\beta_{n} \rightarrow \beta, \alpha_{n}<\beta_{n}$ para todo $n \in \mathbb{N}$ e $\beta_{1}>0$. Tomemos $m_{1}$ e $k_{1}$ os menores inteiros, tais que:

$$
\begin{gathered}
P_{1}+P_{2}+\ldots+P_{m_{1}}>\beta_{1} \geq P_{1}+\ldots+P_{m_{1}-1} \\
P_{1}+\ldots+P_{m_{1}}-Q_{1}-\ldots-Q_{k_{1}}<\alpha_{1} \leq P_{1}+. .+P_{m_{1}}-Q_{1}-\ldots-Q_{k_{1}-1} .
\end{gathered}
$$


Agora, peguemos $m_{2}$ e $k_{2}$ os menores inteiros, tais que:

$$
\begin{gathered}
P_{1}+\ldots+P_{m_{1}}-Q_{1}-\ldots-Q_{k_{1}}+P_{m_{1}+1}+\ldots+P_{m_{2}}>\beta_{2} \\
P_{1}+\ldots+P_{m_{1}}-Q_{1}-\ldots-Q_{k_{1}}+P_{m_{1}+1}+\ldots+P_{m_{2}}-Q_{k_{1}+1}-\ldots-Q_{k_{2}}<\alpha_{2} .
\end{gathered}
$$

A Figura 2 mostra o comportamento das somas parciais. Primeiro, somamos até obter um número maior que $\beta_{1}$, em seguida, subtraímos termos de forma a obter um número menor que $\alpha_{1}$, adicionamos termos novamente até obter um número maior que $\beta_{2}$ e depois diminuímos até encontrar um número menor que $\alpha_{2}$, e assim sucessivamente, sempre pegando o menor $m_{n}$ e $k_{n}$. Isso é possível, pois $\sum P_{n}=+\infty$ e $\sum Q_{n}=+\infty$.

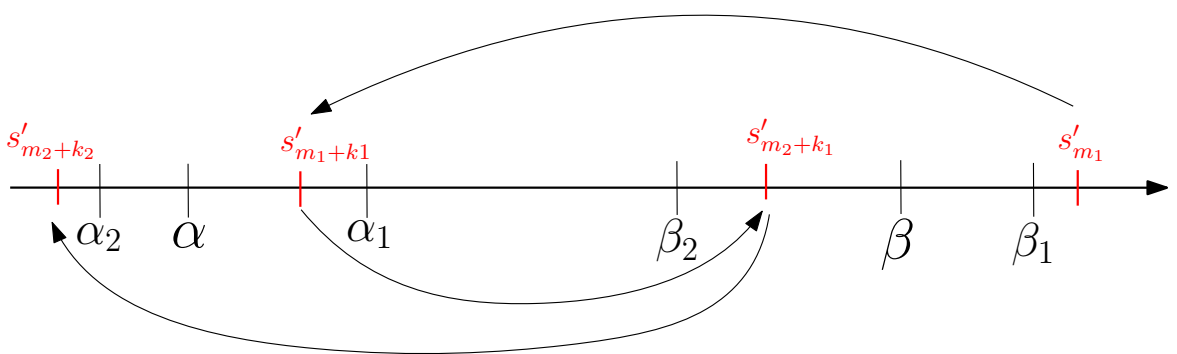

Figura 2: Comportamento das somas parciais.

De forma indutiva, construimos $m_{j}$ e $k_{j}$ os menores inteiros com a propriedade

$$
\begin{gathered}
P_{1}+\ldots+P_{m_{1}}-Q_{1}-\ldots-Q_{k_{1}}+\ldots+P_{m_{j-1}+1}+\ldots+P_{m_{j}}>\beta_{j} \\
P_{1}+\ldots+P_{m_{1}}-Q_{1}-\ldots-Q_{k_{1}}+P_{m_{1}+1}+\ldots+P_{m_{2}}-\ldots-Q_{k_{j-1}+1}-\ldots-Q_{k_{j}}<\alpha_{j} .
\end{gathered}
$$

Se chamarmos de $\left\{s_{n}^{\prime}\right\}$ as somas parciais destes reordenamento, podemos reescrever as desigualdades acima da seguinte forma

$$
s_{m_{j}+k_{j-1}}^{\prime}>\beta_{j} \geq s_{m_{j}+k_{j-1}-1}^{\prime} \text { e } \quad s_{m_{j}+k_{j}}^{\prime}<\alpha_{j} \leq s_{m_{j}+k_{j}-1}^{\prime} .
$$

Deste modo, temos que

$$
0<s_{m_{j}+k_{j-1}}^{\prime}-\beta_{j}<P_{m_{j}} \quad \text { e } \quad 0<\alpha_{j}-s_{m_{j}+k_{j}}^{\prime}<Q_{k_{j}}
$$

A Figura 3 e a Figura 4 ilustram as desigualdades acima. 


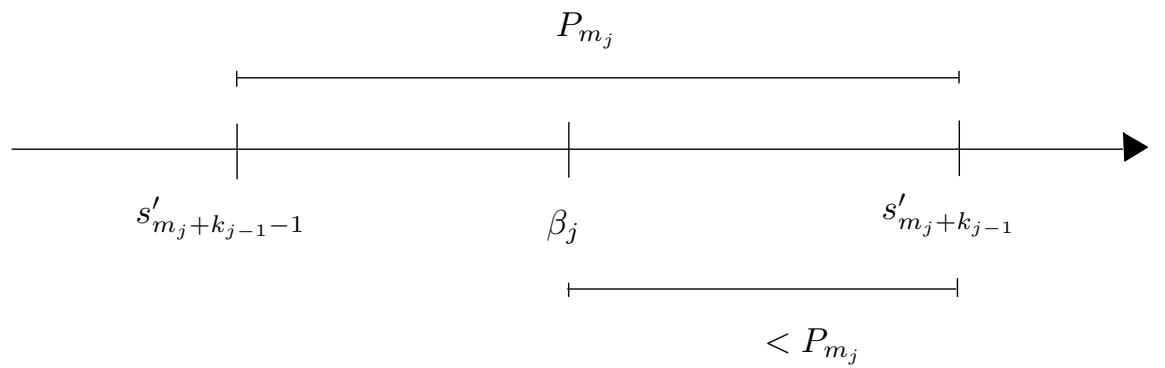

Figura 3: A distância entre $\beta_{j}$ e $s_{m_{j}+k_{j-1}}$ é menor do que $P_{m_{j}}$.

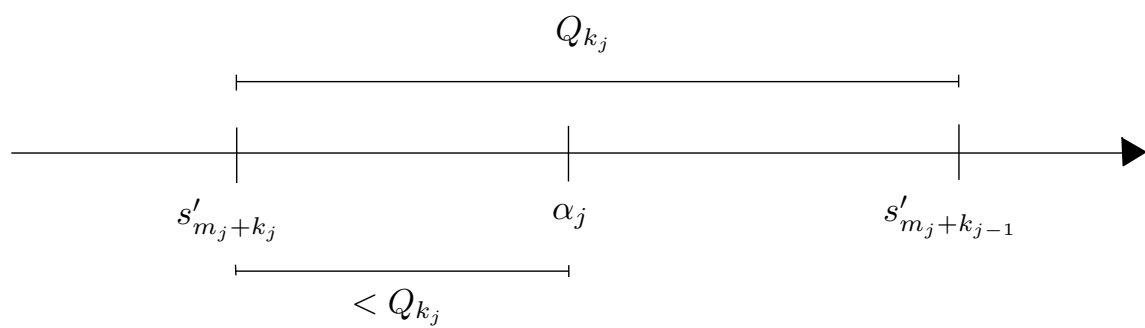

Figura 4: A distância entre $\alpha_{j}$ e $s_{m_{j}+k_{j}}^{\prime}$ é menor do que $Q_{k_{j}}$.

Agora, vamos analizar a relação de $\left\{s_{n}^{\prime}\right\}$ e $\left\{s_{m_{j}+k_{j-1}}\right\}$.

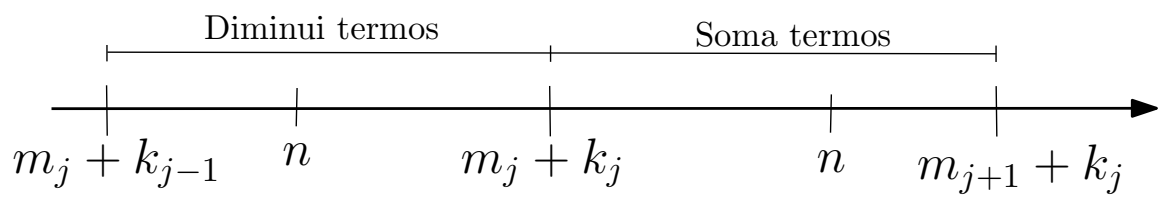

Figura 5: Análise da subsequência $\left\{s_{m_{j}+k_{j-1}}^{\prime}\right\}$.

Se $n \in\left[m_{j}+k_{j-1}, m_{j}+k_{j}\right] \cap \mathbb{N}$, então

$$
s_{n}^{\prime} \leq s_{m_{j}+k_{j-1}}^{\prime} .
$$

Se $\left.n \in] m_{j}+k_{j}, m_{j+1}+k_{j}\right] \cap \mathbb{N}$, então

$$
s_{n}^{\prime} \leq s_{m_{j+1}+k_{j}}^{\prime}
$$

De aqui, se $n \in\left[m_{j}+k_{j-1}, m_{j+1}+k_{j}\right] \cap \mathbb{N}$, então

$$
s_{n}^{\prime} \leq s_{m_{j}+k_{j-1}}^{\prime} \quad \text { ou } \quad s_{n}^{\prime} \leq s_{m_{j+1}+k_{j}}^{\prime} .
$$

Agora, vamos provar que

$\limsup _{n \rightarrow+\infty} s_{n}^{\prime}=\beta \Leftrightarrow\left\{\begin{array}{l}(i) \beta \in E \\ (\text { ii }) \text { Se } x>\beta, \text { então } \exists n_{0} \in \mathbb{N} \text { tal que } s_{n}^{\prime}<x, \forall n \geq n_{0} .\end{array}\right.$ 
Como temos, $P_{m_{j}} \rightarrow 0$, pois $a_{n} \rightarrow 0$, então de (8) segue

$$
\lim _{j \rightarrow+\infty}\left(s_{m_{j}+k_{j-1}}^{\prime}-\beta_{j}\right)=0
$$

e desde que, $\beta_{n} \rightarrow \beta$, temos

$$
\lim _{j \rightarrow+\infty} s_{m_{j}+k_{j-1}}^{\prime}=\lim _{j \rightarrow+\infty}\left[\left(s_{m_{j}+k_{j-1}}^{\prime}-\beta_{j}\right)+\beta_{j}\right]=\beta .
$$

Aqui, temos que $\beta \in E$. Como $\left\{s_{m_{j}+k_{j-1}}^{\prime}\right\} \rightarrow \beta$ e tomamos $x>\beta$, então existe $j_{1} \in \mathbb{N}$ tal que

$$
s_{m_{j}+k_{j-1}}^{\prime}<x, \quad \forall j \geq j_{1} .
$$

Se $n>m_{j_{1}}+k_{j_{1}}$, então existe um $r \in \mathbb{N}$ tal que

$$
m_{j_{r}}+k_{j_{r}-1} \leq n<m_{j_{r}+1}+k_{j_{r}} .
$$

Portanto, por (9) temos que

$$
s_{n}^{\prime} \leq s_{m_{j_{r}}+k_{j_{r}-1}}^{\prime} \text { ou } s_{n}^{\prime} \leq s_{m_{j_{r}+1}+k_{j_{r}}}^{\prime} \quad \Rightarrow \quad s_{n}^{\prime}<x
$$

Com isso, provamos que $\beta=\limsup _{n \rightarrow+\infty} s_{n}^{\prime}$. A prova do $\liminf \inf _{n \rightarrow+\infty} s_{n}^{\prime}=\alpha$ é feito de forma análoga.

\section{Reordenamentos da série harmônica alternante}

Para esta seção precisamos do Teorema de Abel.

Teorema 4.1. (Teorema de Abel) Seja $g(x)=\sum_{n \geq 0} c_{n} x^{n}$ uma série de potência que converge para $|x|<1$. Se $\sum_{n \geq 0} c_{n}$ converge, então

$$
\lim _{x \rightarrow 1^{-}} g(x)=\sum_{n \geq 0} c_{n}
$$

Demonstração. Ver demonstração em [1] ou [9].

Exemplo 4.1. Vamos demonstrar que

$$
\sum \frac{(-1)^{n+1}}{n}=1-\frac{1}{2}+\frac{1}{3}-\frac{1}{4}+\frac{1}{5}-\frac{1}{6}+\frac{1}{7}-\frac{1}{8}+\frac{1}{9}-\frac{1}{10}+\ldots=\ln 2 .
$$


Com efeito, considere

$$
f(x)=x-\frac{1}{2} x^{2}+\frac{1}{3} x^{3}-\frac{1}{4} x^{4}+\frac{1}{5} x^{5}-\frac{1}{6} x^{6}+\ldots=\sum_{n=1}^{+\infty} \frac{(-1)^{n+1}}{n} x^{n}
$$

este é convergente se $|x|<1$. Temos que

$$
f^{\prime}(x)=\sum_{n=1}^{+\infty} \frac{(-1)^{n+1}}{n} n x^{n-1}=\sum_{n=1}^{+\infty}(-1)^{n+1} x^{n-1} .
$$

Multiplicando ambos os lados por $x$, temos

$$
x f^{\prime}(x)=\sum_{n=1}^{+\infty}(-1)^{n+1} x^{n}
$$

Somando (11) e (12), obtemos

$$
x f^{\prime}(x)+f^{\prime}(x)=\sum_{n=1}^{+\infty}(-1)^{n+1} x^{n}+\sum_{n=1}^{+\infty}(-1)^{n+1} x^{n-1}=\sum_{n=1}^{+\infty}\left[(-1)^{n+1} x^{n}-(-1)^{n} x^{n-1}\right] .
$$

Do lado direito da igualdade temos a série telescópica, então

$$
\begin{aligned}
x f^{\prime}(x)+f^{\prime}(x) & =\lim _{k \rightarrow+\infty} \sum_{n=1}^{k}\left[(-1)^{n+1} x^{n}-(-1)^{n} x^{n-1}\right] \\
& =\lim _{k \rightarrow+\infty}\left[(-1)^{k+1} x^{k}-(-1)\right] \\
& =1 .
\end{aligned}
$$

Teremos

$$
f^{\prime}(x)=\frac{1}{1+x}
$$

Então,

$$
f(x)=\int f^{\prime}(x) d x=\int\left(\frac{1}{1+x}\right) d x=\ln (x+1) .
$$

Voltando a (10), temos que

$$
\ln (x+1)=\sum_{n=1}^{+\infty} \frac{(-1)^{n+1}}{n} x^{n}
$$

Agora, aplicando o Teorema de Abel

$$
\sum_{n=1}^{+\infty} \frac{(-1)^{n+1}}{n}=\lim _{x \rightarrow 1^{-}} \ln (x+1)=\ln 2 .
$$


Exemplo 4.2. Será provado que uma reorganização da série $\sum_{n=1}^{+\infty} \frac{(-1)^{n+1}}{n}$ nos leva $a \ln 2+\frac{1}{2} \ln 3$.

Para isto, será seguida a reorganização

$$
1+\frac{1}{3}+\frac{1}{5}-\frac{1}{2}+\frac{1}{7}+\frac{1}{9}+\frac{1}{11}-\frac{1}{4}+\ldots
$$

Por meio desta reorganização obtém-se a série,

$$
f(x)=x+\frac{1}{3} x^{3}+\frac{1}{5} x^{5}-\frac{1}{2} x^{6}+\frac{1}{7} x^{7}+\frac{1}{9} x^{9}+\frac{1}{11} x^{11}-\frac{1}{4} x^{12} \ldots
$$

esta série de potência pode ser vista da seguinte forma

$$
f(x)=\left(x+\frac{1}{3} x^{3}+\frac{1}{5} x^{5}+\frac{1}{7} x^{7}+\frac{1}{9} x^{9}+\ldots\right)-\left(\frac{1}{2} x^{6}+\frac{1}{4} x^{12}+\frac{1}{6} x^{18}+\frac{1}{8} x^{24}+\ldots\right) .
$$

Seja,

$$
g(x)=x+\frac{1}{3} x^{3}+\frac{1}{5} x^{5}+\frac{1}{7} x^{7}+\frac{1}{9} x^{9}+\ldots
$$

$\mathrm{e}$

$$
h(x)=\frac{1}{2} x^{6}+\frac{1}{4} x^{12}+\frac{1}{6} x^{18}+\frac{1}{8} x^{24}+\ldots .
$$

ambas convergem absolutamente no intervalo $-1<x<1$. Como antes, tem-se

$$
\begin{aligned}
g^{\prime}(x) & =1+x^{2}+x^{4}+x^{6}+x^{8}+\ldots \\
& =\frac{1}{1-x^{2}} \\
& =\frac{\frac{1}{2}}{1+x}+\frac{\frac{1}{2}}{1-x} .
\end{aligned}
$$

Integrando, obtem-se

$$
g(x)=\frac{1}{2} \ln (1+x)-\frac{1}{2} \ln (1-x) .
$$

Do mesmo modo,

$$
\begin{aligned}
h^{\prime}(x) & =3 x^{5}+3 x^{11}+3 x^{17}+\ldots \\
& =3 x^{5}\left(1+x^{6}+x^{12}+\ldots\right) \\
& =\frac{3 x^{5}}{1-x^{6}} .
\end{aligned}
$$


Integrando, temos

$$
h(x)=\frac{-1}{2} \ln \left(1-x^{6}\right) .
$$

Como temos que $f(x)=g(x)-h(x)$, então

$$
\begin{aligned}
f(x) & =\frac{1}{2} \ln (1+x)-\frac{1}{2} \ln (1-x)+\frac{1}{2} \ln \left(1-x^{6}\right) \\
& =\frac{1}{2}\left[\ln (1+x)-\ln (1-x)+\ln \left(1-x^{6}\right)\right] \\
& =\frac{1}{2} \ln \left[\frac{(1+x)\left(1-x^{6}\right)}{(1-x)}\right]=\frac{1}{2} \ln \left[(1+x)\left(1+x+x^{2}+x^{3}+x^{4}+x^{5}\right)\right] .
\end{aligned}
$$

Aplicando o Teorema de Abel,

$$
1+\frac{1}{3}+\frac{1}{5}-\frac{1}{2}+\frac{1}{7}+\frac{1}{9}+\frac{1}{11}-\frac{1}{4}+\ldots=\lim _{x \rightarrow 1^{-}} f(x)=\frac{1}{2} \ln 12=\ln 2+\frac{1}{2} \ln 3 .
$$

\section{Conclusão}

Em suma desde o início da nossa aprendizagem da Matemática, sabemos que se considerarmos qualquer conjunto finito de números e reorganizarmos sua ordem, a soma permanecerá a mesma. No entando quando fazemos tal ação com somas infinitas, em alguns casos, podemos reorganizar os termos para obter uma soma totalmente diferente da primeira. Deste modo, isto nos mostra que não devemos confiar apenas na nossa intuição quando trabalhamos com a noção do infinito.

O Teorema de Riemann atribui uma compreensão mais profunda das somas infinitas, fato que intrigou alguns matemáticos durante anos. Riemann foi genial ao perceber que para séries condicionalmente convergentes era possível reorganizar os termos e obter valores diferentes. Por último, este trabalho de Riemann, permite uma maior compreensão da complexidade acerca do tema sobre séries numéricas.

\section{Agradecimentos}

Agradeço ao Orientador deste trabalho em especial, quando ao apresentar o tema possibilitou que eu assim ampliasse os meus conhecimentos na área da Matemática.

\section{Referências}

[1] CONRAD, K.: Boundary behavior of Power series: Abel's theorem. http://www. math. uconn. edu/ kconrad/blurbs/analysis/abelthm.pdf, 2013. 
[2] DIRICHLET, G. L.: Sur la convergence des séries trigonométriques qui servent à représenter une fonction arbitraire entre des limites données. Journal für die reine und angewandte Mathematik, 1829.

[3] DUGUNDJI, J.: Topology. Boston: Allyn and Bacon, INC,1966.

[4] FIGUEIREDO, D.: Análise 1. Rio de Janeiro: LTC, 1996.

[5] GALANOR, S.: Riemann's rearrangement Theorem. National Council of Teachers of Mathematics, 1987.

[6] LEDESMA, G. G. E.: Análise real 1, Notas de aula.

[7] RIEMANN, B.: Uber die Darstellbarkeit einer Function durch eine trigonometrische Reihe. Gesammelte Mathematische Werke, 1867.

[8] RUDIN, W.: Principles of mathematical analysis. New York: McGraw-hill, 1964.

[9] SIQUEIRA, A.: Teorema de Reordenamento de Séries de Riemann e Aplicações, TCC em preparação.

[10] TAUSK, D. V.: Notas Para o Curso de Medida e Integração. 